Archivos de Ciencias de la Educación, Vol. 12, n 13, junio 2018, e046. ISSN 2346-8866 Universidad Nacional de La Plata.

Facultad de Humanidades y Ciencias de la Educación.

Departamento de Ciencias de la Educación.

\title{
Jornada de socialización de proyectos de investigación de la Escuela de Ciencias de la Educación de la Universidad Nacional de Rosario
}

\author{
Pozzo, María Isabel \\ Escuela de Ciencias de la Educación, Universidad Nacional de Rosario, Argentina \\ Ahumada, Adriana \\ Escuela de Ciencias de la Educación, Universidad Nacional de Rosario, Argentina \\ Bisiacco, Florencia \\ Escuela de Ciencias de la Educación, Universidad Nacional de Rosario, Argentina \\ Ordoñez, Lara \\ Escuela de Ciencias de la Educación, Universidad Nacional de Rosario, Argentina \\ Paz, Natalia \\ Escuela de Ciencias de la Educación, Universidad Nacional de Rosario, Argentina \\ Valiente, María Sol \\ Escuela de Ciencias de la Educación, Universidad Nacional de Rosario, Argentina \\ investigaciones.cseduc.unr@gmail.com
}

Cita sugerida: Pozzo, M. I., Ahumada, A., Bisiacco, F., Ordoñez, L., Paz, N. y Valiente, M. S. (2018). [Revisión del libro Jornada de socialización de proyectos de investigación de la Escuela de Ciencias de la Educación de la Universidad Nacional ]. Archivos de Ciencias de la Educación, 12 (13), e046. https://doi.org/10.24215/23468866e046

Recibido: 30 Abril 2018 - Aceptado: 04 Junio 2018 - Publicado: 29 de junio de 2018

(1) (2) Esta obra está bajo licencia Creative Commons Atribución-NoComercial-CompartirIgual 4.0 Internacional cc) 


\title{
Jornada de socialización de proyectos de investigación de la Escuela de Ciencias de la Educación de la Universidad Nacional de Rosario
}

\author{
Maria Isabel Pozzo \\ Escuela de Ciencias de la Educación, Universidad Nacional \\ de Rosario, Argentina \\ Adriana Abumada \\ Escuela de Ciencias de la Educación, Universidad Nacional \\ de Rosario, Argentina \\ Florencia Bisiacco \\ Escuela de Ciencias de la Educación, Universidad Nacional \\ de Rosario, Argentina \\ Lara Ordoñez \\ Escuela de Ciencias de la Educación, Universidad Nacional \\ de Rosario, Argentina \\ Natalia Paz \\ Escuela de Ciencias de la Educación, Universidad Nacional \\ de Rosario, Argentina \\ Maria Sol Valiente \\ Escuela de Ciencias de la Educación, Universidad Nacional \\ de Rosario, Argentina \\ investigaciones.cseduc.unr@gmail.com
}

En 2017, la Escuela de Ciencias de la Educación de la Universidad Nacional de Rosario (UNR) aprobó la creación de un nuevo espacio pensado como ámbito de confluencia de las actividades de investigación de estudiantes, docentes y graduados de las carreras de Ciencias de la Educación y Formación docente que allí se imparten. Se inauguró así el "Espacio de Investigaciones de la Escuela de Ciencias de la Educación”, algunos de cuyos objetivos son:

- Implementar una política institucional acerca de la investigación instalando debates teóricometodológicos al interior de las carreras de la Escuela y entre ellas.

- Promover la práctica de la investigación incentivando la participación de la comunidad educativa en proyectos acreditados en vistas a su capacitación para la investigación.

- Organizar actividades formativas a partir de la detección de demandas del medio educativo colaborando con los distintos espacios, como el taller de Tesina de la carrera de Ciencias de la Educación.

- Contribuir a la realización y difusión de los resultados de prácticas pre-profesionales, trabajos de investigación y/o tesinas en vistas a enriquecer la experiencia colectiva en su proyección a otras instituciones académicas y al medio en general.

- Visualizar áreas de vacancias que promuevan la generación de nuevas líneas de búsqueda, dentro de las cuales la Escuela sea un ámbito y un posible objeto de investigación. (Pozzo, 2017, pp. 2-3) 
Para hacer efectivos los objetivos planteados, se presentaron propuestas de acciones concretas entre las que podemos destacar la organización de actividades de intercambio, difusión y socialización de investigaciones en curso, buscando generar mecanismos de comunicación e información propios de la actividad investigativa.

En ese marco, el pasado 2 de octubre de 2017 se convocó a estudiantes, docentes y graduados a la "Jornada de socialización de los proyectos de investigación de la Escuela de Ciencias de la Educación”. La actividad contó con la concurrencia de los distintos claustros de la institución, como así también con profesionales de otros espacios académicos de distintas facultades e instituciones del país y del extranjero que compartieron los proyectos de investigación en los que trabajan.

Durante la jornada se expusieron doce proyectos. Los disertantes presentaron aportes a la reflexión sobre distintas problemáticas referidas al ámbito de la educación, abordando tópicos específicos que pueden ser consultados en el blog de la Escuela. ${ }^{1}$

Las investigaciones tuvieron como hilo conductor la necesidad de aportar propuestas que mejoren las prácticas educativas y el desarrollo profesional de quienes se encuentran involucrados en la enseñanza.

De los doce trabajos, tres hacen foco en el profesor como profesional; dos de los cuales se centran en la universidad. El primero, denominado "Enseñanza y afiliación institucional en los inicios de la vida universitaria. Una investigación centrada en profesores de primer año", hace referencia a una investigación desarrollada por la Dra. María Paula Pierella con la dirección de la Dra. Sandra Carli y la co-dirección de María Isabel Pozzo. El segundo, titulado "Los procesos de construcción y producción de conocimiento en las carreras de posgrado. El caso de la Maestría en Práctica Docente de la FHyA-UNR”, comparte avances de una investigación dirigida por la Dra. Liliana Sanjurjo. Este trabajo analiza los procesos de construcción y de producción de conocimiento que desarrollan los estudiantes de Maestría en Práctica Docente de la Facultad de Humanidades y Artes de la Universidad Nacional de Rosario. En el tercero, bajo el título "Prácticas profesionales en el asesoramiento curricular", la Prof. Marta Crivelli y equipo indagan sobre el rol de asesor curricular del graduado en Ciencias de la Educación, delineando los futuros campos a transitar.

Otras dos investigaciones, en este caso de la Escuela de Ciencias de la Educación, hacen foco en los estudiantes del nivel secundario y están específicamente orientadas a la inclusión educativa. La primera se desarrolla bajo la dirección de la Prof. María Verónica Zamudio, actual Directora de la Escuela de Ciencias de la Educación, y se titula: "La inclusión socioeducativa en el nivel secundario. Complejidades, tensiones y desafíos en las instituciones educativas". Este trabajo toma como base la Ley de Educación Nacional No 26.206 y las normativas nacionales y provinciales posteriores a 2006, para preguntarse por la inclusión educativa de jóvenes y los discursos que convergen en las instituciones educativas. La segunda aborda la inclusión desde el trabajo con estudiantes de establecimientos educativos de diversos sectores sociales buscando visibilizar todo lo que se pone en juego y se comparte en estos contextos. Se trata de un trabajo, realizado por la Prof. Alejandra Troffe y dirigido por la Dra. Emilia Lebus, denominado: "El encuentro de las diferencias. La escuela más allá de las fronteras del aula”.

En otra línea, centrada en la relación profesor-estudiante y en la constante vinculación del proceso de enseñanza-aprendizaje con el conocimiento, se presentó el proyecto de investigación titulado "Apropiación de conocimientos y cambio subjetivo. Análisis del taller como dispositivo de intervención en educación". Este trabajo, dirigido por Laura Arocena, indaga sobre el modo en que esta modalidad de intervención y su metodología de trabajo impactan en la apropiación de conocimiento de diferentes lenguajes artísticos, corporales y conceptuales, entre otros.

Por otro lado, atendiendo el importante papel que las nuevas tecnologías comenzaron a jugar en diversos campos educativos, se presentaron dos investigaciones. En primer lugar, un proyecto desarrollado por el Prof. Pablo Urbaitel, bajo la dirección de la Prof. Sandra Valdettaro, titulado: "Mediatización, dispositivos móviles y experiencia urbana”. Este trabajo indaga la articulación entre los dispositivos móviles informáticos y las experiencias urbanas (entre las que se destaca la escuela) en el contexto actual de mediatización general. En segundo lugar, la Prof. Susana Copertari dirige la investigación "Formación docente y prácticas universitarias 
a distancia en comunidades virtuales de la UNR”. A través de esta investigación, se indaga la incorporación de plataformas como herramientas de gestión de aprendizaje en las comunidades de las unidades académicas del campus virtual universitario. Específicamente, aquellas que favorezcan prácticas inclusivas a partir de narrativas pedagógicas y relatos de los estudiantes en la Formación Docente.

Abordando otra temática social, se presentó el proyecto "El impacto del modelo socioeconómico agrícola en el legado cultural y la transmisión de la identidad chacarera. Sus efectos en el desarrollo sustentable de la tierra entre 1984 y 2015”, dirigido por la Dra. Natalia Forlini. Este trabajo busca producir, a través de la noción de identidad, un conocimiento explicativo en torno a la configuración de la identidad chacarera. A su vez, tiene como propósito hacer visible los agronegocios que se profundizan en el sur de la provincia de Santa Fe y cómo estos van dejando profundas huellas de diferenciación en los procesos sociales de poblaciones pequeñas.

Desde un abordaje retrospectivo, dos proyectos enmarcados en distintos contextos históricos bucean el pasado en busca de un futuro transformador. El primero, denominado "Arte, pedagogía y cultura en ciudad de Rosario (1930-1950)”, dirigido por la Dra. María Elisa Welti, investiga la singular articulación producida entre artistas, intelectuales y pedagogos en el campo cultural de la ciudad de Rosario entre 1930 y 1950. El segundo, "Pasado y futuro del verbo educar: la pedagogía y el tiempo que vendrá", dirigido por la Dra. María Silvia Serra, busca articular los relatos de los pedagogos modernos y las utopías de su tiempo con las pedagogías actuales, donde surgen horizontes sociales distópicos con un masivo y generalizado descreimiento por lo que vendrá.

Por último, la Dra. María Isabel Pozzo, quien dirige "Cooperación académica internacional e investigación en educación. Un estudio de casos en el nivel superior de Argentina y Brasil”, se refirió al interés por producir conocimientos acerca de los alcances de la cooperación académica internacional para contribuir a la formación profesional en el nivel superior. La investigación toma como caso al Proyecto VISIR+, que involucra carreras de Ingeniería de Brasil (PUC Río, UFSC e IFSC) y la Argentina (UNSE y UNR).

Tras la exposición de cada uno de los proyectos se habilitó un espacio de preguntas y uno mayor al final de la Jornada. De esta manera, los concurrentes tuvieron ocasión de realizar sus aportes generándose así un fluido intercambio de opiniones que se tradujo en la solicitud, por parte del público asistente, de reeditar periódicamente el evento. La jornada puso de manifiesto, una vez más, el interés de la comunidad académica por la investigación educativa.

En estas instancias los estudiantes manifestaron sus inquietudes acerca del tiempo de duración de las investigaciones y de su financiación. Sobre estos puntos los disertantes proporcionaron información referida a aspectos institucionales de los proyectos de investigación tratándose, en algunos casos, de tesis de posgrado en curso, en otros, de proyectos radicados en la misma universidad; y, en unos pocos, de proyectos con financiamiento sustantivo.

El cierre del evento estuvo a cargo de la Coordinadora del Espacio de Investigación de la Escuela de Ciencias de la Educación, Dra. María Isabel Pozzo, quien señaló la importancia de la investigación en la formación profesional como una premisa prácticamente indiscutible en las instituciones universitarias y por ende también en la Escuela de Ciencias de la Educación de la UNR. Resaltó a la investigación educativa como un medio formativo que conjuga creatividad y rigurosidad, poniendo en juego conocimientos disponibles y reclamando otros nuevos. A su vez, en tanto actividad productora de saberes, la investigación educativa se constituye en una herramienta indispensable para el accionar y la toma de decisiones en todos los niveles.

En síntesis, la Jornada permitió avanzar en la consolidación de un nuevo espacio de intercambio y reflexión sobre la enseñanza, con el convencimiento de que no es posible pensar la educación sin el aporte de investigadores comprometidos que contribuyan a comprender su complejidad. 


\section{Bibliografía}

Pozzo, M.I. (2017). Propuesta de creación del Espacio de Investigaciones de la Escuela de Ciencias de la Educación. Universidad Nacional de Rosario.

\section{Notas}

1 Escuela de Ciencias de la Educación, Facultad de Humanidades y Artes, Universidad Nacional de Rosario. Blog: http s://escuelacseducacion.wordpress.com/ 